\title{
Treatment-emergent sexual dysfunction in randomized trials of vortioxetine for major depressive disorder or generalized anxiety disorder: a pooled analysis
}

\author{
Paula L. Jacobsen, ${ }^{*}$ Atul R. Mahableshwarkar, ' William A. Palo, 'Yinzhong Chen, ' \\ Marianne Dragheim, ${ }^{2}$ and Anita H. Clayton ${ }^{3 \dagger}$
}

\author{
${ }^{1}$ Takeda Development Center Americas, Deerfield, Illinois, USA \\ ${ }^{2}$ H. Lundbeck A/S, Copenhagen, Denmark \\ ${ }^{3}$ Department of Psychiatry and Neurobehavioral Sciences, University of Virginia, Charlottesville, Virginia, USA
}

Objective. Antidepressants are frequently associated with treatment-emergent sexual dysfunction (TESD). Vortioxetine, which was approved for patients with major depressive disorder (MDD), has a receptor profile that suggests limited impact on sexual functioning.

Methods. Arizona Sexual Experiences Scale (ASEX) patient-level data were pooled from 7 short-term vortioxetine trials (6 in MDD, 1 in generalized anxiety disorder) and analyzed for incidence of TESD at any post-baseline visit in patients without sexual dysfunction at baseline (defined as ASEX total score $\geq 19$; individual ASEX item score $\geq 5$; or a score $\geq 4$ on any 3 ASEX items). The primary objective was to confirm the non-inferiority of vortioxetine $5-20 \mathrm{mg} /$ day to placebo on the incidence of TESD. Comparisons were based on the common risk difference (95\% confidence interval). Additional analyses compared vortioxetine to duloxetine and duloxetine to placebo. A sensitivity analysis, defined as TESD at 2 consecutive post-baseline visits, was conducted.

Results. TESD incidence, relative to placebo, generally increased with vortioxetine dose with vortioxetine $5 \mathrm{mg}$ non-inferior to placebo. Vortioxetine 10,15 , and $20 \mathrm{mg}$ did not meet the non-inferiority criterion, but no dose had a significantly higher risk of developing TESD versus placebo. Changes in ASEX individual item scores supported the similarity of vortioxetine doses to placebo. Significantly higher TESD risk occurred with duloxetine $60 \mathrm{mg} /$ day versus placebo and versus vortioxetine 5 or $10 \mathrm{mg}$. The sensitivity analysis was generally consistent with the primary analysis. Rates of spontaneously reported sexual adverse events were low.

Conclusions. Vortioxetine was associated with rates of TESD that were not significantly different from placebo in short-term clinical trials.

Received 17 March 2015; Accepted 14 July 2015; First published online 17 November 2015

Key words: Antidepressant, Arizona Sexual Experiences Scale (ASEX), generalized anxiety disorder (GAD), major depressive disorder (MDD), selective serotonin reuptake inhibitors (SSRIs), serotonin norepinephrine reuptake inhibitors (SNRIs), sexual adverse events, vortioxetine.

* Address for correspondence: Paula L. Jacobsen, Takeda Development Center Americas, Inc., One Takeda Parkway, Deerfield, IL 60015, USA. (Email: paula.jacobsen@takeda.com)

$\uparrow$ This study was supported by the Takeda Pharmaceutical Company, Ltd., and H. Lundbeck A/S. Assistance with writing and manuscript preparation was provided by Ken Scholz, PhD, and Philip Sjostedt, BPharm, with The Medicine Group, and was paid for by the Takeda Pharmaceutical Company, Ltd. and H. Lundbeck A/S. The authors are entirely responsible for the scientific content of the paper.

\section{Introduction}

Major depressive disorder (MDD) and generalized anxiety disorder (GAD) are each associated with high rates of sexual dysfunction. One long-term study, for example, estimated that sexual dysfunction was nearly twice as common in people with MDD as in the general population. ${ }^{1}$ Pharmacotherapy for MDD or GAD can 
alleviate sexual dysfunction in some patients, but treatment with most antidepressants is itself associated with some form of sexual dysfunction or worsening sexual dysfunction. $^{2}$ As many as $30 \%-80 \%$ of patients treated with selective-serotonin reuptake inhibitors (SSRIs) experience some form of treatment-emergent sexual dysfunction (TESD), which can affect any aspect of sexual activity, including desire, arousal, and orgasm. ${ }^{2-6}$ Sexual dysfunction associated with antidepressant use reduces self-esteem and quality of life and burdens interpersonal relationships. ${ }^{7,8}$ It is particularly bothersome to patients ${ }^{9}$ and may affect treatment compliance.

The biological substrate underpinning TESD is incompletely understood. Based on multiple studies of SSRIs, it is generally thought that elevated serotonin (5-HT) levels overall exert inhibitory effects on sexual function. ${ }^{10}$ However, preclinical research-and to a lesser extent clinical research-suggests that different 5-HT receptor subtypes may differentially modulate sexual function $\left(\mathrm{eg}\right.$, in male rats, $5-\mathrm{HT}_{1 \mathrm{~A}}$ receptor stimulation is pro-sexual, whereas $5-\mathrm{HT}_{1 \mathrm{~B}}$ receptor stimulation inhibits sexual behavior). ${ }^{11,12}$ Other neurotransmitter systems and their interaction with sex hormones are likely to be involved, including dopaminergic, cholinergic, alphaadrenergic, and nitric oxide systems. ${ }^{10,13}$

The antidepressant vortioxetine has a spectrum of effects on the serotonin system that differs from the profiles of preexisting agents. In vitro evidence suggests that vortioxetine is an antagonist at $5-\mathrm{HT}_{3}, 5-\mathrm{HT}_{7}$, and 5 - $\mathrm{HT}_{1 \mathrm{D}}$ receptors; an agonist at $5-\mathrm{HT}_{1 \mathrm{~A}}$ receptors; a partial agonist at $5-\mathrm{HT}_{1 \mathrm{~B}}$ receptors; and an inhibitor of the $5-\mathrm{HT}$ transporter. ${ }^{14}$ In preclinical studies, vortioxetine affected male rat sexual performance much less than did an SSRI, and it appeared that one or more of vortioxetine's receptor activities counteracted the 5-HT-mediated inhibition of sexual performance produced by serotonin transporter inhibition. ${ }^{15}$ Vortioxetine is an approved antidepressant in the United States, Canada, Europe, and several other countries. The approved therapeutic dose range is $5-20 \mathrm{mg} /$ day with a recommended starting dose of $10 \mathrm{mg}$ /day, after which the dose can be increased or decreased based on individual response. Individual, randomized, placebo-controlled clinical trials have reported TESD incidence rates for lower vortioxetine doses similar to or lower than those seen with placebo; for higher vortioxetine doses, rates were higher, but not significantly so. ${ }^{16}$

\section{Methods}

Seven randomized, placebo-controlled trials of vortioxetine- 6 in patients with $\mathrm{MDD}^{17-22}$ and 1 in patients with $\mathrm{GAD}^{23}$-assessed TESD among participants using the Arizona Sexual Experiences Scale (ASEX) (Table 1). Five of these trials included duloxetine $(60 \mathrm{mg})$, a serotonin- norepinephrine reuptake inhibitor (SNRI), as an active reference. The ASEX is a validated, self-administered patient questionnaire that measures sex drive, ease of arousal, ability to achieve erection (in men) or vaginal lubrication (in women), ease of reaching orgasm, and satisfaction from orgasm. ${ }^{24}$ It was developed to identify and monitor sexual functioning in psychiatric patients in clinical trials. The ASEX consists of 5 questions with responses to each question ranging from 1 to 6 . Higher scores are indicative of worse sexual functioning. There are male and female versions of the ASEX, and patients are encouraged to answer all questions regardless of current frequency of sexual activity.

\section{Aims}

For the current analysis, patient-level ASEX data from all 7 trials that utilized the ASEX were pooled and analyzed to estimate the incidence of TESD during short-term (8 weeks) treatment with vortioxetine compared to placebo or duloxetine. Patients were enrolled into the trials independent of their level of sexual functioning. In order to evaluate the impact of treatment on patients with normal sexual functioning, the primary analysis included only patients without sexual dysfunction at study baseline. The analysis also explored the effects of patient gender and age, as well as vortioxetine dose, on the incidence of TESD. Data from patients who had sexual dysfunction at baseline were also analyzed to assess worsening of sexual dysfunction during treatment. The incidences of spontaneously reported adverse events (AEs) related to sexual functioning were also reported.

\section{Study participants}

The designs of the individual trials have been published previously $^{17-23}$ and will be summarized briefly. For trials in MDD, all patients had to meet the criteria for a major depressive episode (MDE) lasting at least 3 months as described in the Diagnostic and Statistical Manual of Mental Disorders, Fourth Edition, Text Revision (DSM-IV-TR), and be at least 18 years old. Additional inclusion criteria included Montgomery-Åsberg Depression Rating Scale (MADRS) scores of at least 22 (NCT00672620) or at least 26 (all other trials). ${ }^{25}$ Some trials also required Clinical Global Impression-Severity of Illness (CGI-S) scores of at least 4 . For the trial in $\mathrm{GAD}$, participants had to have a primary diagnosis of GAD as defined by DSM-IV-TR, a total score of at least 20 on the Hamilton Anxiety Rating Scale (HAM-A), a score of at least 2 for the HAM-A items of anxious mood and tension, and a score less than or equal to 16 on the MADRS. Trial participants treated with vortioxetine doses of 1 or $2.5 \mathrm{mg}$ per day (below currently approved dosages) were not included in the current analyses. 


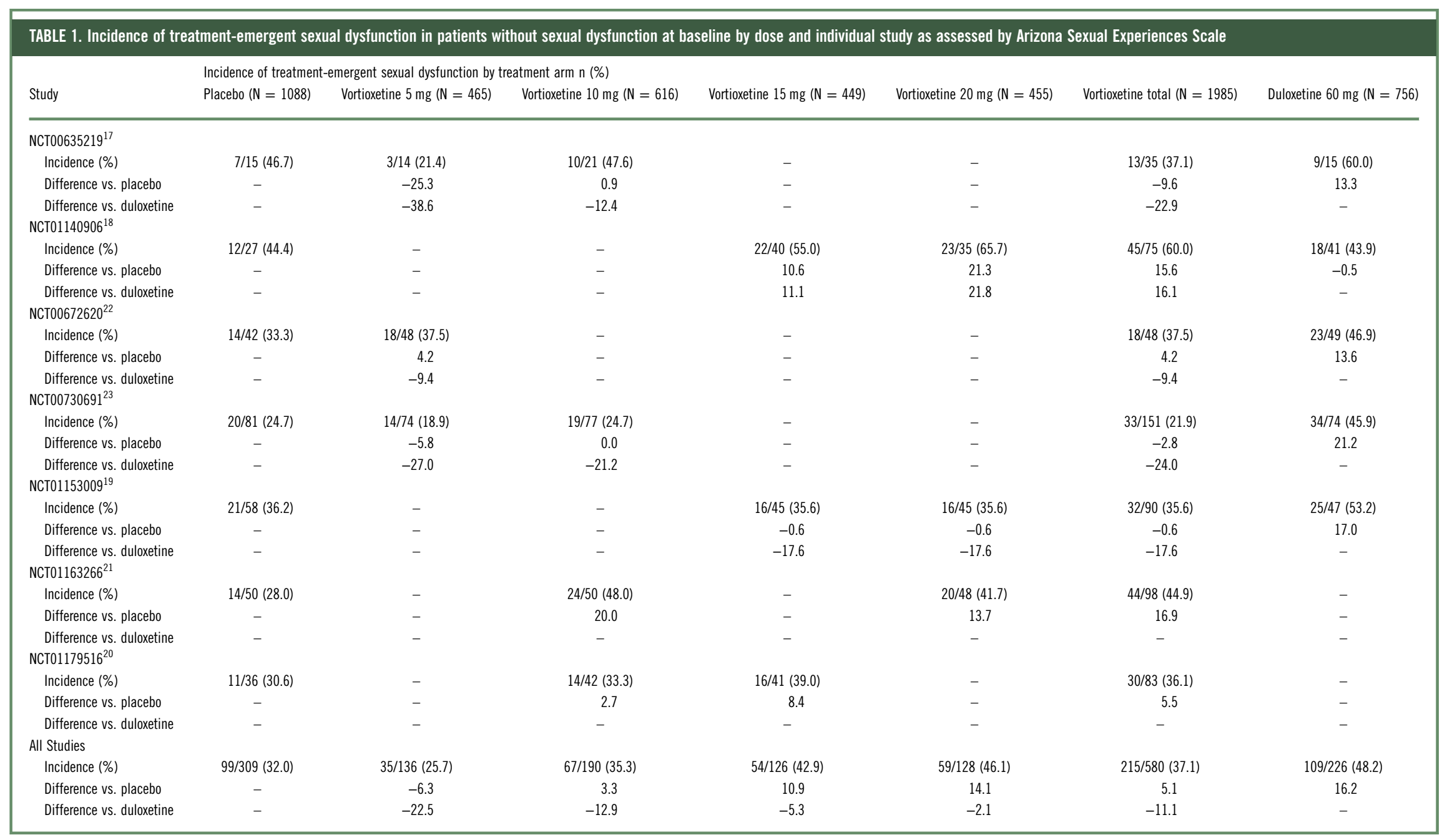


All trials were conducted according to the World Medical Association Declaration of Helsinki, the ICH Harmonised Tripartite Guidelines for Good Clinical Practice, and all applicable local or regional regulatory requirements. An ethics committee for each country or site approved the protocol, and written informed consent was obtained from each participant before any study procedures were performed. All trials were registered with ClinicalTrials.gov (Table 1).

\section{Main outcome measures}

The prespecified primary objective of the analysis was to compare vortioxetine to placebo for the risk of TESD via a non-inferiority hypothesis in patients without sexual dysfunction at baseline. Sexual dysfunction at baseline or at any clinic visit was defined as an ASEX total score of at least 19, a score of at least 5 on any ASEX individual item, or a score of at least 4 on any 3 ASEX individual items. ${ }^{24}$ TESD was defined as sexual dysfunction (meeting the above criteria) at any time during the treatment period. Trial participants with sexual dysfunction at baseline were excluded from the primary analysis (see below for additional analysis of worsening sexual dysfunction).

The comparison of each vortioxetine dose $(5,10,15$, and $20 \mathrm{mg}$ /day) to placebo for the primary objective was reported using a $95 \%$ confidence interval (CI) for the common risk difference in TESD incidence between each dose and placebo. The risk difference of the TESD incidence between each dose and placebo was calculated (percent vortioxetine minus percent placebo). The common risk difference for the set of observed differences in the incidence of TESD between each vortioxetine dose and placebo was estimated using the Cochran-Mantel-Haenszel (CMH) method stratified by study. ${ }^{26}$ Non-inferiority of vortioxetine versus placebo was evaluated by comparing the upper bound of the 95\% CI for the common risk difference with a clinically meaningful margin of $10 \%$, with non-inferiority declared if the upper bound was less than the $10 \%$ margin.

An additional analysis was conducted to investigate if vortioxetine resulted in significant reductions in developing TESD compared to duloxetine via a superiority hypothesis in trials where vortioxetine and duloxetine were studied together. The risk difference of each vortioxetine dose compared to duloxetine was estimated (percent vortioxetine minus percent duloxetine), and comparisons based on the common risk difference were performed using the same approach as for the comparisons between each dose and placebo. The superiority of vortioxetine versus duloxetine was declared if the upper bound of the $95 \%$ CI was less than zero. Comparisons between duloxetine and placebo were also conducted (percent duloxetine minus percent placebo) to investigate whether duloxetine resulted in increased
TESD compared to placebo. Subgroup analyses were also performed to explore the effects of patient age and gender on the risk of developing TESD.

Sensitivity analyses were conducted to more thoroughly evaluate the changes in ASEX scores during treatment. Analyses included defining TESD at 2 consecutive study visits in patients without sexual dysfunction at baseline and at least 2 post-baseline visits, and analyzing the change from baseline at each study visit in ASEX individual item scores for all patients with a baseline score and at least one post-baseline score. The analysis method of TESD at 2 consecutive study visits was similar to that for TESD in the primary analysis. The analysis of the change from baseline to each study visit in the ASEX individual item scores was performed using a mixed model for repeated measures (MMRM) with treatment, study, week, treatment-by-week interaction, and baseline score-by-week as fixed effects. Comparisons of the difference between each vortioxetine dose and placebo (or duloxetine) were reported using $95 \%$ CIs using observed data with a focus on the changes from baseline to week 8 .

Subjects identified as having sexual dysfunction at study baseline could not contribute to the evaluation of TESD in the primary analysis, but were evaluated to identify possible worsening of their condition. Worsening was defined as a shift of at least 3 different ASEX individual items at the same visit, with a shift defined as the change of an item score from at most 3 to greater than 3 , or from 4 to ( 5 or 6 ), or from 5 to 6 during the treatment period. The data were analyzed using the same method as the primary analysis to test for non-inferiority of vortioxetine versus placebo and for superiority of vortioxetine versus duloxetine.

All comparisons were conducted without adjustment for multiple comparisons and using a 0.05 level of significance. In addition, spontaneously reported AEs related to sexual dysfunction were pooled from 14 randomized, placebo-controlled, short-term $(6$ or 8 weeks) trials of vortioxetine in patients with MDD or GAD (see Appendix 2) and were summarized by gender for each treatment group. The list of AEs related to sexual dysfunction was predefined based on Medical Dictionary for Regulatory Activities (MedDRA) v14.1 and is provided in Appendix 1.

\section{Findings}

TESD results from the 7 trials included in the analysis are provided in Table 1. Of the 3829 treated patients, 1115 were without sexual dysfunction at baseline and were considered candidates for developing TESD during the treatment period. The percentage of patients without sexual dysfunction at baseline was similar across treatment arms (placebo, $309 / 1088$ [28.4\%]; all doses of vortioxetine combined, 580/1985 [29.2\%]; duloxetine, 226/756 [29.9\%]). 
Among the dose groups, the incidences of TESD with $5 \mathrm{mg}(25.7 \%)$ were lower than placebo $(32.0 \%)$; with $10 \mathrm{mg}(35.3 \%)$, they were similar to placebo; and with $15 \mathrm{mg}$ and $20 \mathrm{mg}$ (42.9\% and $46.1 \%$, respectively), they were higher than placebo. However, overall, the incidence of TESD in vortioxetine-treated groups combined $(37.1 \%)$ was not significantly different from placebo and was lower than with duloxetine $(48.2 \%)$.

\section{Risk for developing TESD}

Figure 1 shows the common risk difference (95\% CI) for developing TESD at any visit as assessed by the ASEX for vortioxetine and duloxetine versus placebo across the 7 pooled trials. The risk of developing TESD in the $5 \mathrm{mg}$ vortioxetine group was lower than in the placebo group (common risk difference, $-4.6 \%$ ) and non-inferior to placebo (upper bound of the $95 \%$ CI for the common risk difference was less than $10 \%$ ). The other 3 vortioxetine doses did not meet the criteria for non-inferiority to placebo; however, none of the doses had a significantly higher risk than placebo. The risk of TESD in the duloxetine group was significantly higher than the placebo group (lower bound of the $95 \%$ CI was greater than 0). Although not shown in Figure 1, the risk of TESD in the duloxetine-treated group was also significantly higher than in the 5 and $10 \mathrm{mg}$ vortioxetine groups (common risk difference $[95 \%$ CI $]$ was 22.0 [10.6-33.5] for duloxetine versus $5 \mathrm{mg}$ vortioxetine and 19.6 [5.7-33.5] for duloxetine versus $10 \mathrm{mg}$ vortioxetine) but not significantly higher than in the 15 and $20 \mathrm{mg}$ vortioxetine groups $(4.2$ [-10.8-19.1] for duloxetine versus $15 \mathrm{mg}$ vortioxetine and -0.2 [-15.3-15.0] for duloxetine versus $20 \mathrm{mg}$ vortioxetine).

For men, the risk of TESD associated with $5 \mathrm{mg}$ vortioxetine was non-inferior to and lower than placebo. Other vortioxetine doses did not satisfy the noninferiority criterion, but did not have a significantly higher risk than placebo. In contrast, the TESD risk associated with duloxetine was significantly greater than with placebo. For women, none of the vortioxetine doses met the criterion for non-inferiority to placebo, but as for men, the risk for TESD was not significantly greater than with placebo. For women, the risk associated with duloxetine was also not significantly greater than with placebo, but it was significantly higher than with $5 \mathrm{mg}$ vortioxetine (19.1\% [2.2-35.9]) and $10 \mathrm{mg}$ vortioxetine (26.3\% [6.4-46.2]).

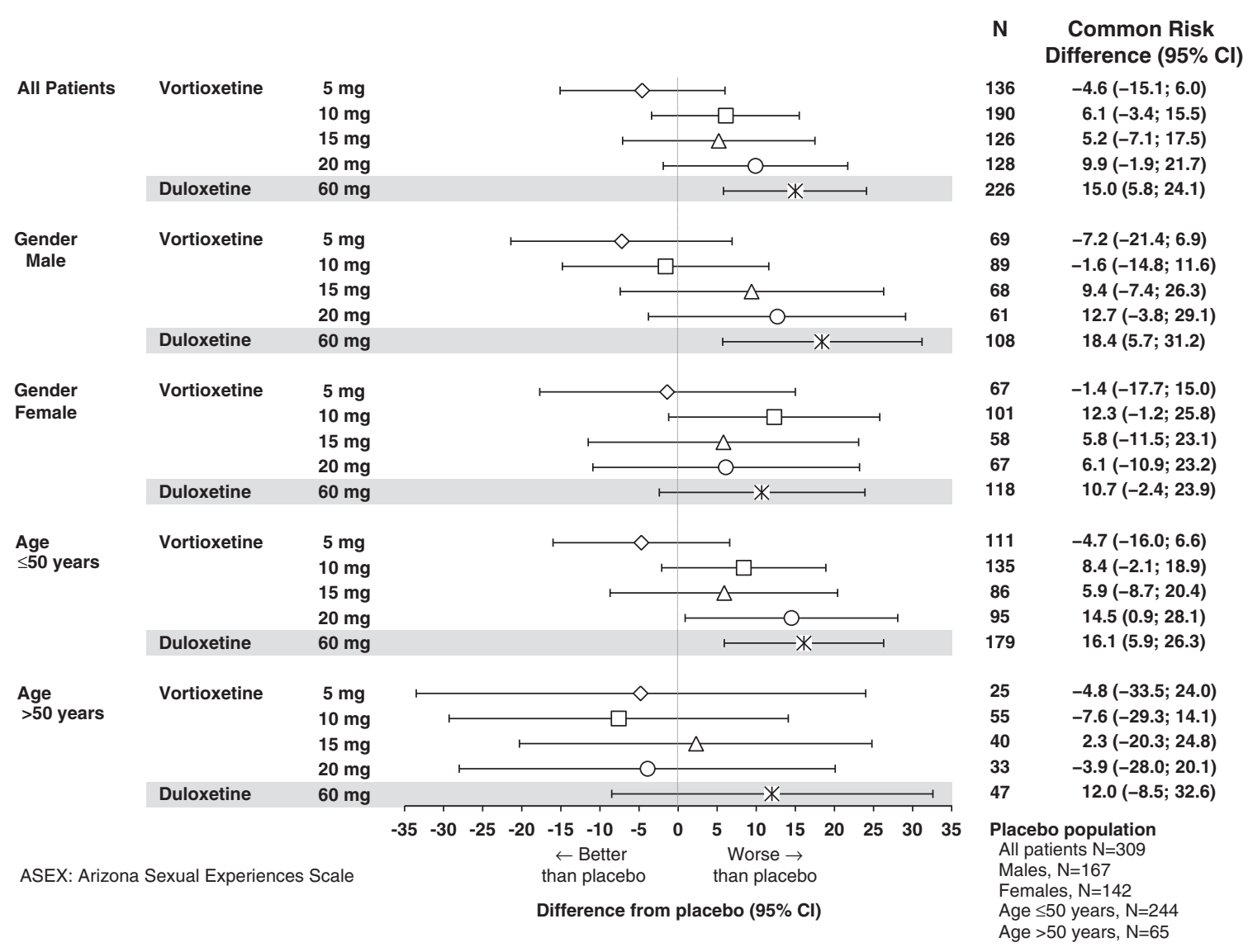

FIGURE 1. Common risk difference of developing treatment-emergent sexual dysfunction in patients without sexual dysfunction at baseline in the major depressive disorder/generalized anxiety disorder short-term data pool as assessed by ASEX. 
In patients 50 years or younger, $5 \mathrm{mg}$ vortioxetine was non-inferior to placebo in TESD risk, and the risk with the $20 \mathrm{mg}$ vortioxetine dose was significantly greater than with placebo. The risk for TESD associated with duloxetine was also significantly greater than with placebo, as well as significantly greater than with $5 \mathrm{mg}$ vortioxetine dose (17.6\% [5.3-29.9]). In patients older than 50 years, no vortioxetine dose was associated with a risk for TESD that was non-inferior to, or significantly higher than, the risk with placebo. However, the number of patients in this subgroup was small, and the risk associated with duloxetine was also not significantly greater than that of placebo. In addition, the risk associated with duloxetine was also not significantly greater than that of placebo. However, the number of patients in this subgroup was small, so caution should be taken not to draw conclusions regarding less TESD in the $>50$ age group based on this analysis.

\section{Sensitivity analyses}

The pooled incidence of TESD at 2 consecutive clinic visits was $16.5 \%(49 / 297)$ in the placebo group; $18.9 \%$ (25/132), 21.7\% (39/180), 25.8\% (32/124), and 31.7\%
(40/126) in the $5,10,15$, and $20 \mathrm{mg}$ vortioxetine groups, respectively; and $27.4 \%(57 / 208)$ in the duloxetine group. The risk for TESD at 2 consecutive clinic visits in all vortioxetine groups and in the duloxetine group, based on the common risk difference, was numerically higher than in the placebo group; however, no vortioxetine dose had a risk significantly higher than that of placebo (Figure 2). Although the incidence of TESD at 2 consecutive visits for the duloxetine group was less than for the $20 \mathrm{mg}$ vortioxetine group, the risk in the duloxetine group was significantly greater than with placebo due to the smaller CI. There were no significant differences between any vortioxetine dose and duloxetine. In subgroups defined by gender and age, the common risk differences were generally consistent with the overall analysis and primary analysis for TESD at any visit. For men, the risk of TESD associated with duloxetine was significantly higher than with placebo, and for patients aged 50 years or younger, the risk was higher for duloxetine and vortioxetine $20 \mathrm{mg}$ compared to placebo. No differences were seen between groups for women and for those older than 50 years.

The second sensitivity analysis examined the changes from baseline to week 8 (end of the treatment period) in

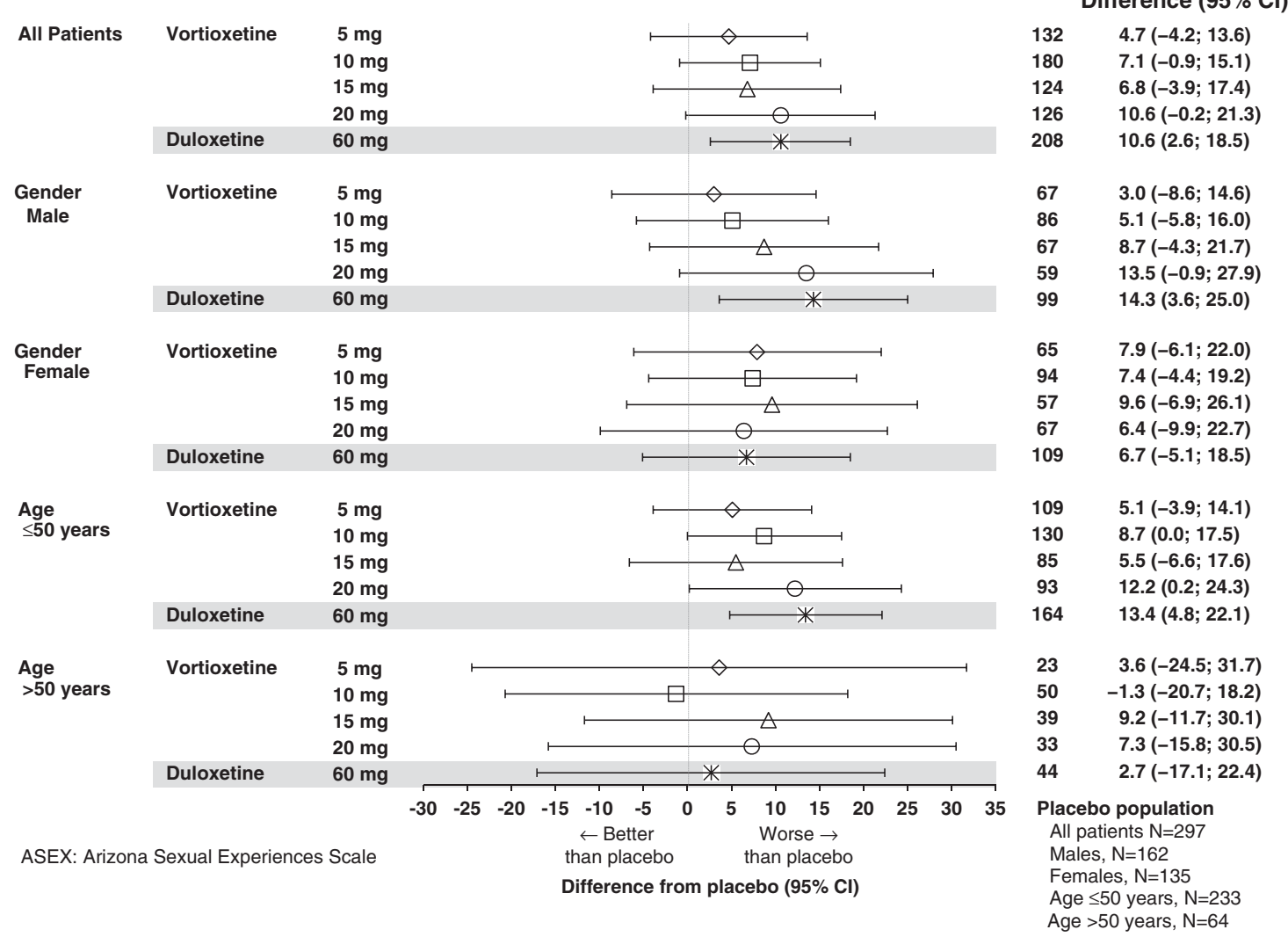

FIGURE 2. Common risk difference of developing treatment-emergent sexual dysfunction at 2 consecutive visits in patients without sexual dysfunction at baseline in the major depressive disorder/generalized anxiety disorder short-term data pool as assessed by ASEX. 


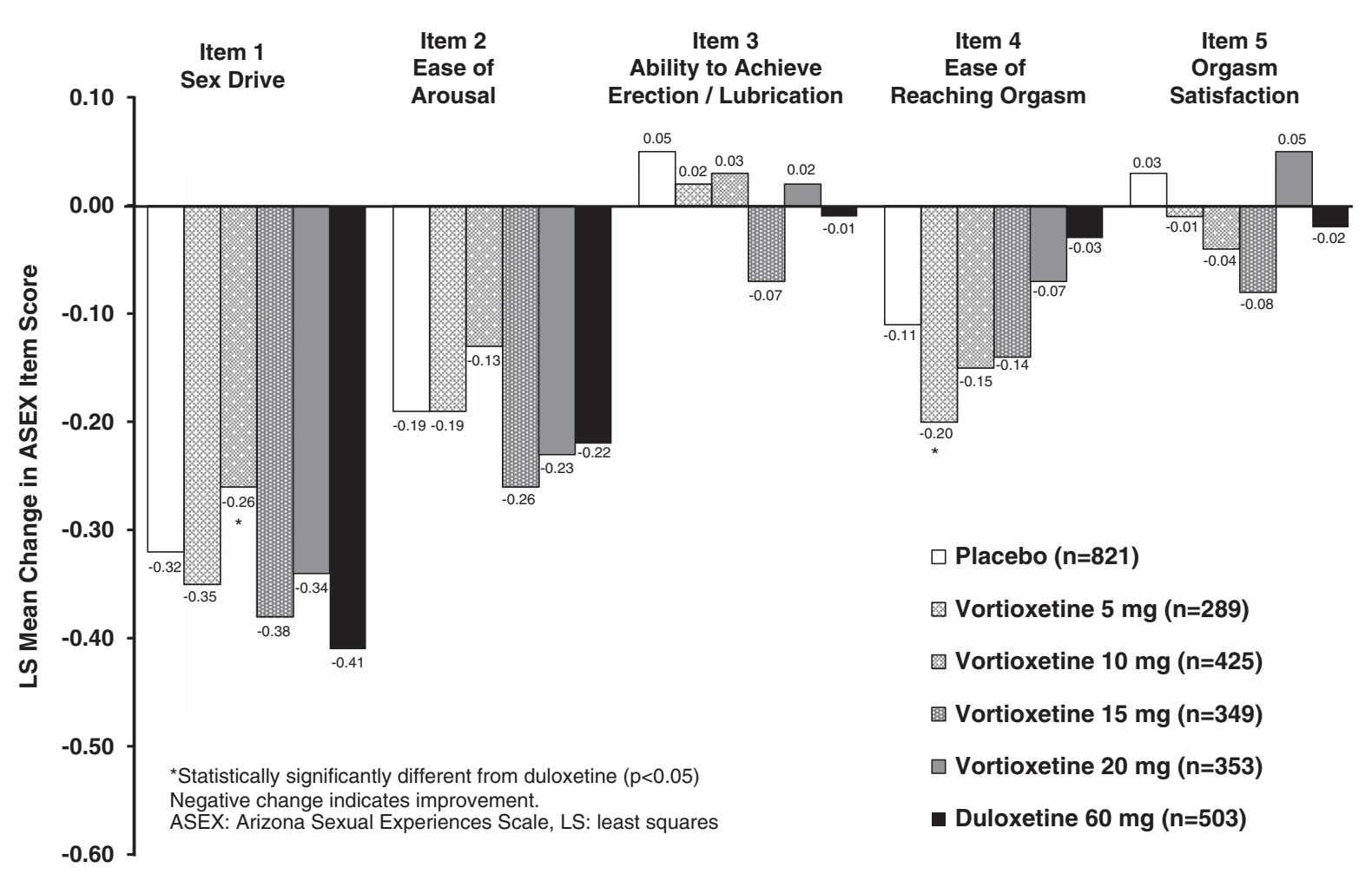

FIGURE 3. LS mean change in ASEX individual item scores from baseline to week 8 in the major depressive disorder/generalized anxiety disorder short-term data pool.

ASEX individual item scores (Figure 3) for all patients. Patients in all treatment arms (including placebo) exhibited mean improvements in ASEX individual item scores for items 1 (sex drive), 2 (ease of arousal), and 4 (ease of reaching orgasm). Mean changes from baseline in items 3 (ability to achieve erection/lubrication) and 5 (orgasm satisfaction) were small and inconsistent. Consistent with the primary analysis, changes from baseline in ASEX individual item scores in the vortioxetinetreated groups were generally similar to those in the placebo group. There were no significant differences between any of the vortioxetine dose groups or duloxetine and placebo in ASEX individual item scores.

\section{Risk for worsening sexual dysfunction}

The effects of vortioxetine therapy in patients with preexisting sexual dysfunction at baseline were evaluated by analysis of the risk of worsening sexual dysfunction (Figure 4). Overall, the risk of worsening sexual dysfunction associated with vortioxetine treatment was similar to that associated with placebo. The 5 and $15 \mathrm{mg}$ vortioxetine groups were non-inferior to placebo in this regard. In the 10 and $20 \mathrm{mg}$ vortioxetine groups, the risk was higher than for placebo, but the differences were not significant. Duloxetine was associated with significantly higher risk of worsening compared to placebo (common risk difference $7.7 \%$ [0.6-14.9]) and to $5 \mathrm{mg}$ vortioxetine (common risk difference $12.5 \%$ [3.3-21.7]). In the subgroups defined by gender and age, there were no clear trends differing from the overall results.

\section{Spontaneously reported adverse events related to sexual dysfunction}

The analysis of spontaneously-reported AEs related to sexual dysfunction included results from 14 short-term (6 or 8 weeks) (see Appendix 2), randomized, controlled trials of vortioxetine in patients with MDD or GAD based on a predefined search list of MedDRA preferred terms. In total, 3377 patients received vortioxetine $(5-20 \mathrm{mg} /$ day) in these trials. The results are shown in Table 2. Overall, $23 / 2230(1.0 \%)$ patients who received placebo reported at least one $\mathrm{AE}$ related to sexual dysfunction. The corresponding numbers in all vortioxetine- and duloxetine-treated patients were $74 / 3377(2.2 \%)$ and $52 / 907(5.7 \%)$, respectively, with the incidence of sexual AEs similar across all 4 vortioxetine doses. The most common AEs were decreased libido, anorgasmia, and abnormal orgasm in both vortioxetine and placebo groups. The incidence of sexual AEs was generally higher for men than for women for all treatment groups (including placebo). For women, the most frequently reported sexual dysfunction $\mathrm{AE}$ was related to libido (loss 


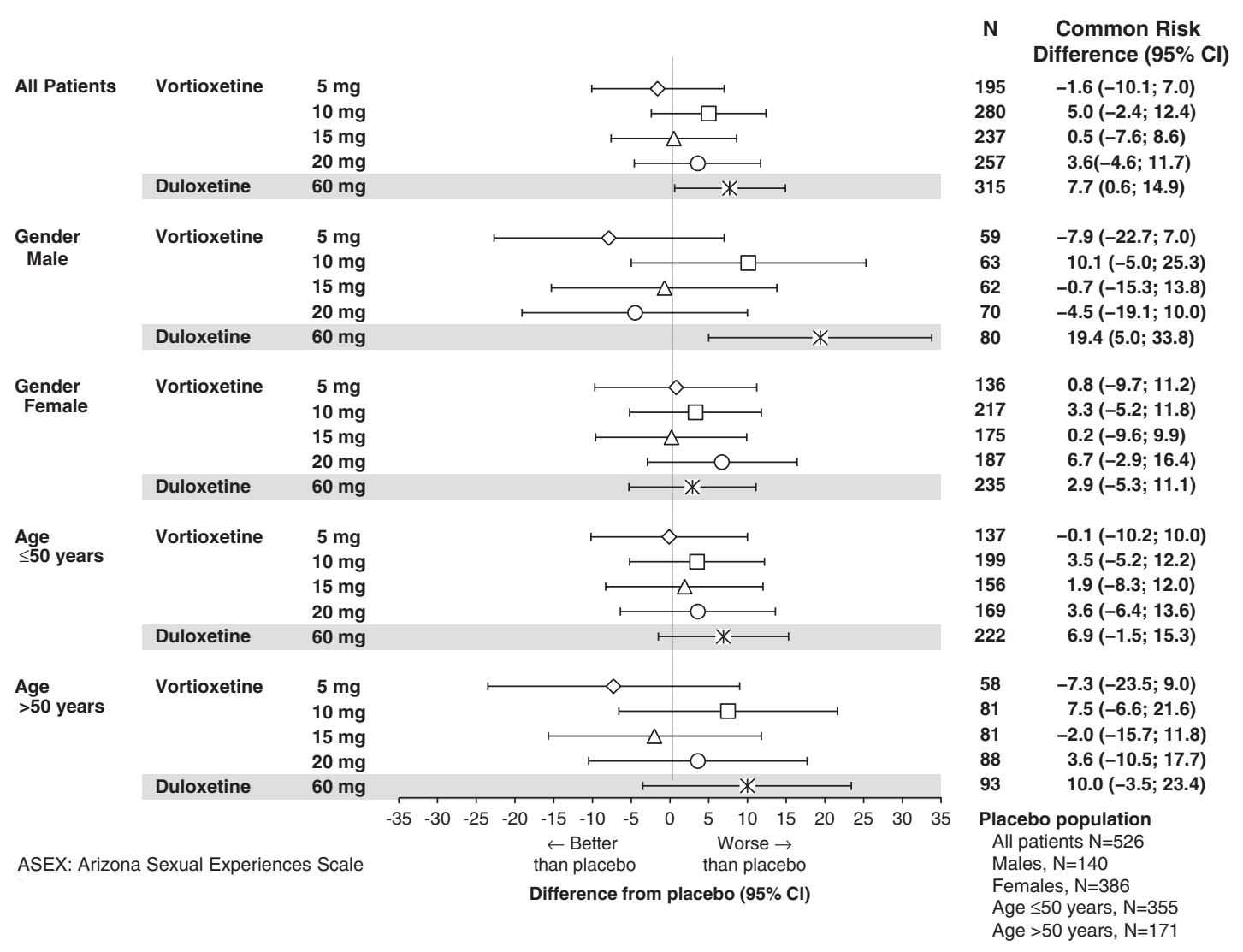

FIGURE 4. Common risk difference of worsening of sexual dysfunction in patients with sexual dysfunction at baseline in the major depressive disorder/ generalized anxiety disorder short-term data pool as assessed by ASEX.

or decreased). For men, the most commonly reported sexual dysfunction AEs were problems with ejaculation and erectile dysfunction. There was no consistent trend toward an increased incidence of AEs with increasing doses of vortioxetine. The incidence of spontaneously reported AEs was higher in the duloxetine-treated group than in the vortioxetine or placebo groups.

Four patients receiving vortioxetine discontinued therapy because of an $\mathrm{AE}$ related to sexual dysfunction: 2 in the $5 \mathrm{mg}$ group (decreased libido in 1 patient and concurrent erectile dysfunction and loss of libido in 1 patient), 1 patient in the $10 \mathrm{mg}$ group (sexual dysfunction), and 1 patient in the $20 \mathrm{mg}$ group (anorgasmia). One patient in the duloxetine group discontinued because of an AE related to sexual dysfunction (erectile dysfunction).

\section{Discussion}

The use of validated measures to assess longitudinal shift has been acknowledged as the most appropriate method for the evaluation of changes in sexual function, especially in comparison to the prevalence assessment of sexual dysfunction in observational studies through spontaneous reporting of AEs. Although the evaluation of a shift in sexual functioning is traditionally based on any single visit and does not require confirmation from additional visits, the methodology is seen as superior to the spontaneous reporting of sexual AEs, where patients under-report such AEs unless directly questioned. ${ }^{4,27,28}$ The vortioxetine clinical development program is now the largest clinical database prospectively assessing TESD using a structured scale that longitudinally investigates the shift in sexual dysfunction in patients without any sexual dysfunction at baseline, as well as the worsening of symptoms in those patients with sexual dysfunction at baseline.

Across the 6 randomized, placebo-controlled trials for the treatment of MDD and the single trial for the treatment of GAD, vortioxetine $5-20 \mathrm{mg}$ (the approved therapeutic dose range) was associated with an approximately $5 \%$ increase in incidence of TESD (as determined by ASEX scores) over placebo in patients without sexual dysfunction at baseline. For patients treated with $5 \mathrm{mg}$ vortioxetine, the risk of TESD was non-inferior to the risk observed in patients who received placebo. While the risk of developing TESD was generally similar to, or less than, placebo at 5 and $10 \mathrm{mg}$, and higher than placebo at 15 and $20 \mathrm{mg}$, it generally increased with 


\begin{tabular}{|c|c|c|c|c|c|c|c|c|c|c|c|c|c|c|}
\hline \multicolumn{15}{|l|}{ 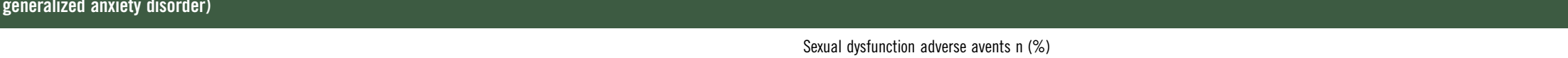 } \\
\hline & \multicolumn{2}{|c|}{$\begin{array}{c}\text { Placebo } \\
(\mathrm{N}=2230)\end{array}$} & \multicolumn{2}{|c|}{$\begin{array}{l}\text { Vortioxetine } 5 \mathrm{mg} \\
\qquad(\mathrm{N}=1466)\end{array}$} & \multicolumn{2}{|c|}{$\begin{array}{l}\text { Vortioxetine } 10 \mathrm{mg} \\
\qquad(\mathrm{N}=1007)\end{array}$} & \multicolumn{2}{|c|}{$\begin{array}{l}\text { Vortioxetine } 15 \mathrm{mg} \\
\quad(\mathrm{N}=449)\end{array}$} & \multicolumn{2}{|c|}{$\begin{array}{l}\text { Vortioxetine } 20 \mathrm{mg} \\
\quad(\mathrm{N}=455)\end{array}$} & \multicolumn{2}{|c|}{$\begin{array}{l}\text { Vortioxetine total } \\
\qquad(\mathrm{N}=3377)\end{array}$} & \multicolumn{2}{|c|}{$\begin{array}{l}\text { Duloxetine } 60 \mathrm{mg} \\
\qquad(\mathrm{N}=907)\end{array}$} \\
\hline Incidence of any sexual adverse event, $\mathrm{N}$ (\%) & \multicolumn{2}{|c|}{$23(1.0)$} & \multicolumn{2}{|c|}{$30(2.0)$} & \multicolumn{2}{|c|}{$25(2.5)$} & \multicolumn{2}{|c|}{$7(1.6)$} & \multicolumn{2}{|c|}{$12(2.6)$} & \multicolumn{2}{|c|}{$74(2.2)$} & \multicolumn{2}{|c|}{$52(5.7)$} \\
\hline Incidence of sexual adverse events by & Males & Females & Males & Females & Males & Females & Males & Females & Males & Females & Males & Females & Males & Females \\
\hline gender, N (\%) & $\mathrm{N}=789$ & $N=1441$ & $N=506$ & $\mathrm{~N}=960$ & $N=326$ & $\mathrm{~N}=681$ & $\mathrm{~N}=141$ & $\mathrm{~N}=308$ & $\mathrm{~N}=143$ & $\mathrm{~N}=312$ & $N=1116$ & $N=2261$ & $\mathrm{~N}=281$ & $\mathrm{~N}=626$ \\
\hline Any sexual adverse event & $13(1.6)$ & $10(0.7)$ & $18(3.6)$ & $12(1.3)$ & $14(4.3)$ & $11(1.6)$ & $5(3.5)$ & $2(0.6)$ & $7(4.9)$ & $5(1.6)$ & $44(3.9)$ & $30(1.3)$ & $40(14.2)$ & $12(1.9)$ \\
\hline Libido decreased & $8(1.0)$ & $6(0.4)$ & $8(1.6)$ & $7(0.7)$ & $4(1.2)$ & $6(0.9)$ & $4(2.8)$ & $1(0.3)$ & $2(1.4)$ & $5(1.6)$ & $18(1.6)$ & $19(0.8)$ & $15(5.3)$ & $2(0.3)$ \\
\hline Orgasm abnormal & $1(0.1)$ & $3(0.2)$ & $2(0.4)$ & $2(0.2)$ & $2(0.6)$ & $3(0.4)$ & $1(0.7)$ & $1(0.3)$ & $2(1.4)$ & $1(0.3)$ & $7(0.6)$ & $7(0.3)$ & $9(3.2)$ & $2(0.3)$ \\
\hline Anorgasmia & 0 & $1(<0.1)$ & $1(0.2)$ & $2(0.2)$ & $2(0.6)$ & $3(0.4)$ & $1(0.7)$ & 0 & $1(0.7)$ & $1(0.3)$ & $5(0.4)$ & $6(0.3)$ & $3(1.1)$ & $7(1.1)$ \\
\hline Ejaculation delayed & $2(0.3)$ & 0 & $2(0.4)$ & 0 & $3(0.9)$ & 0 & 0 & 0 & $1(0.7)$ & 0 & $6(0.5)$ & 0 & $11(3.9)$ & 0 \\
\hline Loss of libido & 0 & 0 & $3(0.6)$ & $1(0.1)$ & $1(0.3)$ & $1(0.1)$ & 0 & 0 & 0 & 0 & $4(0.4)$ & $2(<0.1)$ & 0 & $1(0.2)$ \\
\hline Erectile dysfunction & $3(0.4)$ & - & $2(0.4)$ & - & $3(0.9)$ & - & 0 & - & 0 & - & $5(0.4)$ & - & $13(4.6)$ & - \\
\hline Vulvovaginal dryness & - & 0 & - & 0 & - & $3(0.4)$ & - & 0 & - & 0 & - & $3(0.1)$ & - & 0 \\
\hline Ejaculation disorder & 0 & - & $1(0.2)$ & - & 0 & - & 0 & - & $1(0.7)$ & - & $2(0.2)$ & - & $2(0.7)$ & - \\
\hline Sexual dysfunction & $1(0.1)$ & 0 & 0 & 0 & $1(0.3)$ & 0 & $1(0.7)$ & 0 & 0 & 0 & $2(0.2)$ & 0 & $1(0.4)$ & $1(0.2)$ \\
\hline Disturbance in sexual arousal & 0 & 0 & 0 & $1(0.1)$ & 0 & 0 & 0 & 0 & 0 & 0 & 0 & $1(<0.1)$ & 0 & 0 \\
\hline Orgasmic sensation decreased & 0 & $1(<0.1)$ & 0 & 0 & 0 & 0 & 0 & $1(0.3)$ & 0 & 0 & 0 & $1(<0.1)$ & 0 & 0 \\
\hline Ejaculation failure & $1(0.1)$ & 0 & 0 & 0 & 0 & 0 & 0 & 0 & 0 & 0 & 0 & 0 & $2(0.7)$ & 0 \\
\hline
\end{tabular}


increasing doses of vortioxetine; this pooled analysis indicated, however, that no individual dose of vortioxetine was associated with a significantly higher risk of TESD when compared to placebo. These results are supported by statistical difference between vortioxetine and duloxetine on the 2 sensitivity analyses, which explored the risk of TESD occurring on 2 consecutive study visits during the treatment period and also changes from baseline in ASEX individual item scores.

Previous studies comparing duloxetine to SSRIs (paroxetine $^{29}$ and escitalopram ${ }^{30}$ ) have demonstrated an intermediate effect of duloxetine on sexual functioning between placebo and SSRI therapy, suggesting that duloxetine is a more rigorous comparator agent than an SSRI in the detection of assay sensitivity for sexual dysfunction. In this pooled analysis, assay sensitivity was demonstrated by the significantly higher risk of developing TESD with duloxetine than with placebo. In addition, the risk of TESD with duloxetine was significantly higher than with placebo in all other analyses except ASEX individual item scores. The risk of TESD associated with duloxetine was significantly higher than for low doses of vortioxetine (5 and $10 \mathrm{mg}$ ) in the primary analysis and some of the secondary and subgroup analyses. Overall, the results support the conclusion that therapeutic doses of vortioxetine ( 5 to $20 \mathrm{mg}$ /day) have limited impact on sexual functioning, with some increase in sexual dysfunction at the higher doses. Additional analyses that explored the worsening of sexual dysfunction in patients with sexual dysfunction at baseline also supported the conclusion that vortioxetine has limited effects on sexual function in patients with MDD or GAD.

Similarly, the incidence of spontaneously reported AEs related to sexual dysfunction in the 14 short-term trials (11 in MDD and 3 in GAD, see Appendix 2) were only slightly higher in the vortioxetine-treated patients compared to placebo-treated patients, while duloxetinetreated patients reported higher rates for almost every type of $\mathrm{AE}$ related to sexual dysfunction. As expected, spontaneous reporting of sexual dysfunction is much lower for all treatment groups, including duloxetine, than the rates of sexual dysfunction reported using a prospective questionnaire.

\section{Study limitations}

This pooled analysis primarily consisted of patients with MDD, with a smaller number with GAD; differences in types of and reasons for sexual dysfunction due to the different disease states are not known. However, in the primary and additional analyses, data from $1 \mathrm{GAD}$ trial were pooled with data from $6 \mathrm{MDD}$ trials because the single GAD trial was too small to allow a meaningful analysis of sexual dysfunction beyond that already published. $^{23}$
The high rates of sexual dysfunction at baseline $(\backsim 70 \%)$ limited the number of subjects available for the evaluation of TESD across the vortioxetine clinical trial program. These numbers were further reduced in the subgroup analyses. While change from baseline in ASEX individual item scores represents the only way to assess specific phases of sexual function in studies utilizing the ASEX, this method of analysis of the ASEX has not yet been validated. In this pooled analysis, spontaneous reports of TESD were very low, and the effect of prospective administration of a validated questionnaire on spontaneous reports of sexual dysfunction is unknown. However, the comparisons across treatment groups are consistent with the between-group relationships seen using the ASEX. Also, patients with comorbid psychiatric diseases were excluded from these studies, as were patients receiving concomitant psychotropics, which would limit the generalization of these results to such patients.

The interpretation of this study is further limited because these data were collected from different clinical studies. The individual studies and pooled analysis were not formally powered for testing the statistical hypotheses, and no adjustments were made to control for multiple comparisons. Adequately powered trials are needed to confirm the results, as well as further investigate any dose relationship regarding the incidence of TESD with vortioxetine. Additionally, some of the subgroups included small sample sizes (eg, $>50$ age group), and therefore the interpretation of the results in these cases is limited. Estimation of the treatment effects was limited due to the trial designs and to the ASEX scale only being utilized in 7 of the 14 short-term MDD and GAD trials. Placebo was studied in all 7 trials, duloxetine was studied in 5 of the 7 trials, and not all vortioxetine doses were studied in all trials; therefore, some treatment comparisons (eg, vortioxetine $20 \mathrm{mg}$ versus duloxetine) were based on results from as few as 2 trials.

\section{Conclusions}

Therapeutic doses of vortioxetine (5 to $20 \mathrm{mg} /$ day) had only minor effects on sexual function in patients with MDD or GAD. While the incidence of TESD relative to placebo generally increased with increasing vortioxetine dose in patients without sexual dysfunction at baseline, the effects of vortioxetine on sexual function in these patients did not significantly differ from the effects of placebo. Duloxetine treatment was associated with rates of TESD that were significantly higher than for placebo and significantly higher than for vortioxetine 5 and $10 \mathrm{mg}$.

\section{Disclosures}

Paula L. Jacobsen, William Palo, and Yinzhong Chen are employees of Takeda Development Center Americas. 
Atul R. Mahableshwarkar is an employee of Takeda Development Center and holds stocks of GlaxoSmithKline, Johnson \& Johnson, and Pfizer. Marianne Dragheim is an employee of H. Lundbeck A/S. Anita H. Clayton has received grants from Auspex Pharmaceuticals, Forest Research Institute, Inc., Palatin Technologies, Takeda, and Trimel Biopharma; advisory/consultant fees from Arbor Scientia, Euthymics, Forest Laboratories, Lundbeck, Naurex, Otsuka, Palatin Technologies, Pfizer, Inc., Roche, S1 Biopharmaceuticals, Inc., Sprout Pharmaceuticals, and Takeda Global Research \& Development; has royalties/ copyright with Ballantine Books/Random House, Changes in Sexual Functioning Questionnaire, and Guilford Publications; and holds shares/restricted stock units with Euthymics and S1 Biopharmaceuticals, Inc.

\section{SUPPLEMENTARY MATERIAL}

To view supplementary material for this article, please visit http://dx.doi.org/10.1017/S1092852915000553.

\section{REFERENCES:}

1. Angst J. Sexual problems in healthy and depressed persons. Int Clin Psychopharmacol. 1998; 13(Suppl 6): S1-S4.

2. Kennedy SH, Rizvi S. Sexual dysfunction, depression, and the impact of antidepressants. J Clin Psychopharmacol. 2009; 29(2): 157-164.

3. Montejo AL, Llorca G, Izquierdo JA, Rico-Villademoros F. Incidence of sexual dysfunction associated with antidepressant agents: a prospective multicenter study of 1022 outpatients. Spanish Working Group for the Study of Psychotropic-Related Sexual Dysfunction. J Clin Psychiatry. 2001; 62(Suppl 3): 10-21.

4. Clayton AH, Pradko JF, Croft HA, et al. Prevalence of sexual dysfunction among newer antidepressants. J Clin Psychiatry. 2002; 63(4): 357-366.

5. Serretti A, Chiesa A. Treatment-emergent sexual dysfunction related to antidepressants: a meta-analysis. J Clin Psychopharmacol. 2009; 29(3): 259-266.

6. Williams VS, Edin HM, Hogue SL, Fehnel SE, Baldwin DS. Prevalence and impact of antidepressant-associated sexual dysfunction in three European countries: replication in a cross-sectional patient survey. J Psychopharmacol. 2010; 24(4): 489-496.

7. Baldwin DS, Palazzo MC, Masdrakis VG. Reduced treatmentemergent sexual dysfunction as a potential target in the development of new antidepressants. Depress Res Treat. 2013; 2013: 256841.

8. Reichenpfader U, Gartlehner G, Morgan LC, et al. Sexual dysfunction associated with second-generation antidepressants in patients with major depressive disorder: results from a systematic review with network meta-analysis. Drug Saf. 2014; 37(1): 19-31.

9. $\mathrm{Hu} \mathrm{XH}$, Bull SA, Hunkeler EM, et al. Incidence and duration of side effects and those rated as bothersome with selective serotonin reuptake inhibitor treatment for depression: patient report versus physician estimate. J Clin Psychiatry. 2004; 65(7): 959-965.

10. Graf H, Walter M, Metzger CD, Abler B. Antidepressant-related sexual dysfunction-perspectives from neuroimaging. Pharmacol Biochem Behav. 2014; 121: 138-145.
11. Olivier B, Chan JS, Snoeren EM, et al. Differences in sexual behaviour in male and female rodents: role of serotonin. Curr Top Behav Neurosci. 2011; 8: 15-36.

12. Fabre LF, Clayton AH, Smith LC, Goldstein I, Derogatis LR. The effect of gepirone-ER in the treatment of sexual dysfunction in depressed men. J Sex Med. 2012; 9(3): 821-829.

13. Keltner NL, McAfee KM, Taylor CL. Mechanisms and treatments of SSRI-induced sexual dysfunction. Perspect Psychiatr Care. 2002; 38(3): 111-116.

14. Bang-Andersen B, Ruhland T, Jorgensen M, et al. Discovery of 1-[2-(2,4-dimethylphenylsulfanyl)phenyl]piperazine (Lu AA21004): a novel multimodal compound for the treatment of major depressive disorder. J Med Chem. 2011; 54(9): 3206-3221.

15. Oosting RS, Chan JSW, Pehrson A, Li Y, Sanchez C, Olivier B. P-42-016 Vortioxetine does not affect sexual behavior in a male rat model for sexual dysfunction [abstract]. Int J Neuropsychopharmacol. $2014 ; 17$ (S1): 138.

16. Katona C, Katona C. New generation multi-modal antidepressants: focus on vortioxetine for major depressive disorder. Neuropsychiatr Dis Treat. 2014; 10: 349-354.

17. Baldwin DS, Loft H, Dragheim M. A randomised, double-blind, placebo controlled, duloxetine-referenced, fixed-dose study of three dosages of Lu AA21004 in acute treatment of major depressive disorder (MDD). Eur Neuropsychopharmacol. 2012; 22(7): 482-491.

18. Boulenger J-P, Loft H, Olsen CK. Efficacy and safety of vortioxetine (Lu AA21004), 15 and 20mg/day: a randomized, double-blind, placebo-controlled, duloxetine-referenced study in the acute treatment of adult patients with major depressive disorder. Int Clin Psychopharmacol. 2014; 29(3): 138-149.

19. Mahableshwarkar A, Jacobsen P, Chen Y, Serenko M, Trivedi M. A randomized, double-blind, duloxetine-referenced study comparing efficacy and tolerability of 2 fixed doses of vortioxetine in the acute treatment of adults with MDD. Psychopharmacology (Berl). 2015; 232(12): 2061-2070.

20. Mahableshwarkar A, Jacobsen P, Serenko M, Chen Y, Trivedi M. A randomized, double-blind, placebo-controlled study of the efficacy and safety of 2 doses of vortioxetine in adults with major depressive disorder. J Clin Psychiatry. 2015; 76(5): 583-591.

21. Jacobsen P, Mahableshwarkar A, Serenko M, Chan S, Trivedi M. A randomized, double-blind, placebo-controlled study of the efficacy and safety of vortioxetine $10 \mathrm{mg}$ and $20 \mathrm{mg}$ in adults with major depressive disorder. J Clin Psychiatry. 2015; 76(5): 575-582.

22. Mahableshwarkar AR, Jacobsen PL, Chen Y. A randomized, doubleblind trial of $2.5 \mathrm{mg}$ and $5 \mathrm{mg}$ vortioxetine ( $\mathrm{Lu} \mathrm{AA21004)}$ versus placebo for 8 weeks in adults with major depressive disorder. Curr Med Res Opin. 2013; 29(3): 217-226.

23. Mahableshwarkar AR, Jacobsen PL, Chen Y, Simon JS. A randomised, double-blind, placebo-controlled, duloxetinereferenced study of the efficacy and tolerability of vortioxetine in the acute treatment of adults with generalised anxiety disorder. Int J Clin Pract. $2014 ;$ 68(1): 49-59.

24. McGahuey CA, Gelenberg AJ, Laukes CA, et al. The Arizona Sexual Experience Scale (ASEX): reliability and validity. J Sex Marital Ther. 2000; 26(1): 25-40.

25. American Psychiatric Association (APA). Diagnostic and Statistical Manual of Mental Disorders, 4th ed., text rev.. Washington, DC: American Psychiatric Association; 2000.

26. Sánchez-Meca J, Marín-Martínez F. Meta-analysis of $2 \times 2$ tables: estimating a common risk difference. Educational and Psychological Measurement. 2001; 61(2): 249-276.

27. Reviewer Guidance-Conducting a Clinical Safety Review of a New Product Application and Preparing a Report on the Review. 2005. http://www.fda.gov/downloads/Drugs/ 
GuidanceComplianceRegulatoryInformation/Guidances/ucm 072974.pdf. Accessed July 2, 2014.

28. Landen M, Hogberg P, Thase ME. Incidence of sexual side effects in refractory depression during treatment with citalopram or paroxetine. J Clin Psychiatry. 2005; 66(1): 100-106.

29. Detke MJ, Wiltse CG, Mallinckrodt CH, McNamara RK, Demitrack MA, Bitter I. Duloxetine in the acute and long-term treatment of major depressive disorder: a placebo- and paroxetinecontrolled trial. Eur Neuropsychopharmacol. 2004; 14(6): 457-470.

30. Clayton A, Kornstein S, Prakash A, Mallinckrodt C, Wohlreich M. Changes in sexual functioning associated with duloxetine, escitalopram, and placebo in the treatment of patients with major depressive disorder. J Sex Med. 2007; 4(4 Pt 1): 917-929. 